\title{
$\begin{array}{lllllllllllllll}F & E & A & \boldsymbol{I} & \boldsymbol{U} & \boldsymbol{R} & \boldsymbol{E} & & \boldsymbol{A} & \boldsymbol{R} & \boldsymbol{T} & \boldsymbol{I} & \boldsymbol{C} & \boldsymbol{L} & \boldsymbol{E}\end{array}$
}

\section{Space Charge Measurements on Impregnated Paper: A Review of the PEA Method and a Discussion of Results}

Key Words: Pulsed electrcoacoustic, paper insulation, HVDC, submarine cable

by

Peter Morshuis and Marc Jeroense

Delft University of Technology

\section{Quantitative knowledge about space charge inside electrical insulation is important in order to evaluate the electrical field.}

\section{INTRODUCTION}

lectrical insulation in high voltage equipment is subject - to electrical stress. Severely stressed insulation will age, and in the case of bad material and/or design, may fail in service. To design equipment, it is therefore important to have quantitative knowledge about the electrical field inside the insulation. When the geometry of the insulation, the permittivity of the different materials, and the service voltage $U$ are known, the ac field can be computed with the use of suitable software. Space charge is hardly present in equipment used under ac stress and therefore plays no role of importance.

In equipment being serviced under de stress however, space charge is often present. This charge greatly influences the electrical field. Quantitative knowledge about the presence of space charge is therefore needed. This paper describes the quest for useful measurement principles. One measurement principle, the Pulsed Electroacoustic method, will be described extensively. The method of calibration and the importance of knowledge about the frequency response of the system are described. The results of space charge measurements on impregnated paper samples are presented and discussed, along with a model that predicts the observations fairly well.

\section{Historical Review}

As the number of applications of dc equipment grew (HVDC cables, X-ray systems, televisions, etc.) [1-3], there was a need for quantitative measurement of space charge inside the dielectrics used inside the equipment. Following the history of charge measurement, one recognizes two classes of techniques: destructive and non-destructive techniques.

\section{Destructive Techniques}

The first attempts to quantify charges in and on dielectrics at the beginning of this century were made by using powders. The dielectric was first cut into slabs. After that, charge polarity sensitive powder was put on the slabs. Red-colored lead oxide $\left(\mathrm{Pb}_{3} \mathrm{O}_{4}\right)$ was used to detect positive charges; yellow-white colored sulphur $(S)$ was used to detect negative charges $[2,4,5,6]$. The powders "stuck" to the surface charges on the slabs. Putting the two-dimensional slabs on top of each other revealed the original three-dimensional space charge profile. The disadvantages are evident: the method of preparing the slabs is destructive to the dielectric, the preparation can affect the charge distribution, and only a qualitative knowledge is gained.

Later on, quantitative measurements could be performed by using a field mill or, better, a capacitive (electrostatic) probe $[2,6,7]$. Again, the dielectric had to be cut into slices. The probe approached the charged surface to a certain distance. The induced voltage at the probe was then measured by using a stable, high impedance meter. Although the measurement was quantitative, the previously mentioned disadvantages still remained.

Other techniques, such as TSC (Thermally Stimulated Current), TSSP (Thermally Stimulated Surface Potential) 
and TL (Thermoluminescence) are also used concerning the charge phenomena in dielectrics.

\section{Non-Destructive Techniques}

As the need for non-destructive, quantitative measurement techniques grew, new measurement principles were developed. Most of the measurement principles were developed in the past one or two decades. They will be reviewed briefly.

The TSM (Thermal Shock Method or Thermal Step Method) was the first non-destructive, quantitative method that saw the light of day [8]. First, a flat sample is made. Thin electrodes are then deposited on both sides of the sample. One side of the dielectric sample is exposed to a temperature pulse or step. This thermal pulse or step travels as a thermal wave through the sample. Any space charges inside the sample will be slightly displaced as the thermal wave passes by. This slight displacement of charges results in a simultaneous shift in the electrode charges, which can be measured as a current or voltage (depending on the detection circuit). The propagation of the thermal wave through the sample can be calculated. Together with the voltage or current signal, the original space charge distribution can be calculated using convolution techniques. The disadvantage of this method is the need for rigorous mathematical treatment and the great expense of the equipment.

The PWP (Pressure Wave Propagation) method was a better alternative, and it is widely used nowadays $[2,9,10]$. The principle is very much like that of TSM. Instead of a thermal pulse, the sample is exposed to a pressure pulse of very short duration (one often talks about an acoustic pulse instead of a pressure pulse). The pressure pulse travels as a wave through the sample, displacing any apparent charges inside the dielectric. As with TSM, this results in simultaneous displacement of electrode charges that can be detected as a small voltage or current. Due to the short duration of the pulse, there is no need for convolution techniques to recover the charge distribution. This is an advantage over TSM. A piezoelectric foil, PVDF (Polyvinylidenefluoride), is used as a pressure generating device. However, in the beginning of the development of the PWP method, the pressure pulse was generated by ceramic piezoelectric devices. These methods are known as PPP (Piezoelectrically Generated Pressure Pulse) method and PPS (Piezoelectrically Generated Pressure Step) method.

The LIPP (Laser-Induced Pressure Pulse) method uses the same principle as the PWP method but differs in the generating of the pressure pulse [2,11]. A very short laser pulse $(<<1 \mathrm{~ns})$ irradiates one side of the sample. The material generates a pulsed acoustic wave as a result of the energy shock at the surface of the sample. Like the PWP method, the LIPP method in principle needs no mathematical treatment. The only disadvantage is the rather costly system. The advantage over PWP is the shorter rise time of the stimulating pressure pulse. The decay time, however, is of the same order of mag-

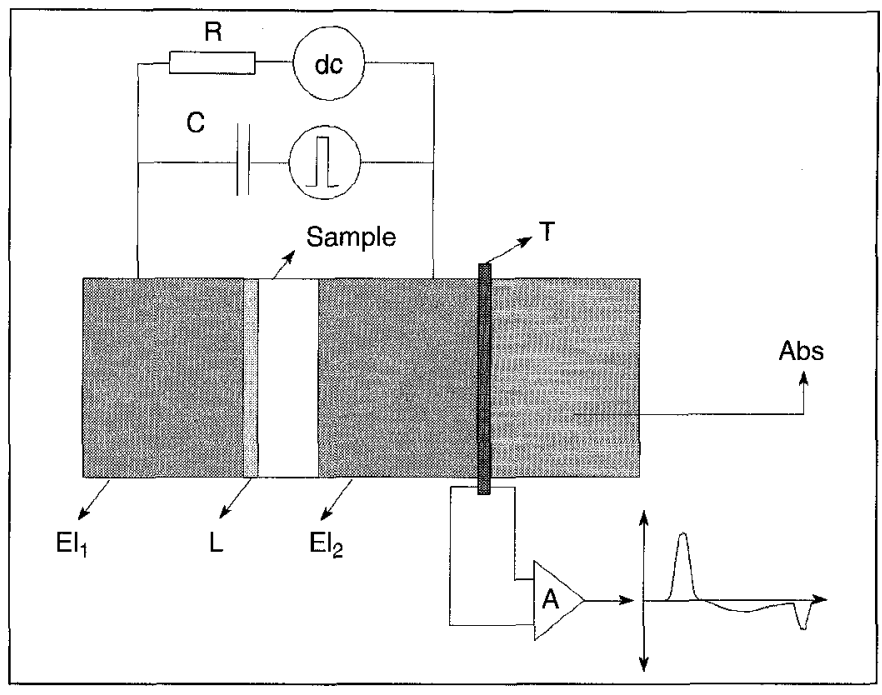

Fig. 1 Circuit description of the PEA measurement system

nitude. In the literature, the names "LIPP" and "PWP" are often used interchangeably.

All of the non-destructive methods named so far use an externally generated wave quantity (thermal or acoustic/pressure) that travels through the whole sample and interacts with the charges when passing by. The principle way of detection is electrical.

The PEA (Pulsed Electroacoustic) method principally differs from the previous mentioned methods $[1,2,12,13,14]$. The method was developed by the research group of Prof. T. Takada (Japan) in close cooperation with Prof. C.M. Cooke (USA). A short electrical pulse is put across the sample. As a result, the charges are stimulated by the pulsed applied field and experience a pulsed force that travels as acoustic waves (from each charge) through the sample. The acoustic waves are detected by a piezoelectric transducer (PVDF foil) that converts them into electric signals. With the PEA method, the wave quantity (acoustic/pressure) is generated at the site of the charges itself. The principle way of detection is acoustic. The method is also known as the ESAW (Electrically Stimulated Acoustic Wave) method.

\section{Detalleo Description of the Pea Method}

\section{Measurement Set-Up}

The principle of the Pulsed Electroacoustic method will be further explained using Fig. 1. The dielectric sample is put between two electrodes. Seen from left to right, the system consists of electrode $E l_{1}$, conducting acoustic layer $L$, the sample, electrode $E l_{2}$, piezoelectric transducer $T$, and acoustic absorber $A b s$. An amplifier is placed across the transducer $T$ that leads its signal to an oscilloscope. A pulse source and a dc source are mounted in parallel across electrodes $E l_{1}$ and $E l_{2}$, using a coupling capacitor $C$ and a protecting resistor $R$. Typical values for the dc voltage are $5-40 \mathrm{kV}$, depending on the sample thickness. Typical values for the pulse voltage and pulse width are $0.1-2 \mathrm{kV}$ and 5-200 ns, respectively. 
The result of putting a de voltage across the sample is two-fold. First, charges are injected in the sample; that is, space charges are generated. Second, electrode charges are formed at the electrodes.

The effect of the pressure pulse is the generation of pressure waves at three principle sites: at electrode $E l_{1}$, at $E l_{2}$ (the electrode charges) and at places inside the sample where space charges have accumulated. They all simultaneously travel through the sample in two directions.

To the right, they travel through the sample and are transferred into electrode $E l_{2}$ (aluminum). This electrode delays the arrival of the acoustic pulse until the disturbances, caused by the firing of the pulse source, have died away. Hereafter, the acoustic wave is transmitted to transducer $T$ (PVDF, typically 4 to 110 :m thick; the smaller, the better resolution). Leaving transducer $T$, the acoustic wave is transmitted into acoustic absorber $A b s$ which delays and suppresses the reflections of the acoustic wave back into transducer $T$. To the left, the acoustic waves first meet the conducting acoustic layer $L$. The need for this layer will be explained later. From here, the acoustic wave is transmitted to electrode $E l_{1}$ (aluminum), after which it will reflect against the air/aluminum interface and will travel back into the system. As all the waves that started traveling to the left will arrive later at transducer $T$ than the waves that started traveling to the right, no overlapping of signals occurs.

The transducer $T$ transforms the acoustic wave into an electrical signal. This signal is amplified by a broadband amplifier and displayed on an oscilloscope.

Reflection and transmission will occur at every acoustic interface. This cannot be ignored and will be discussed later.

\section{Electrostatic Description}

Starting from Poisson's equation for quasi-static fields in a dielectric of constant relative permittivity $\varepsilon_{r}$, given by

$$
\nabla \cdot E=\frac{\rho}{\varepsilon_{0} \varepsilon_{r}},
$$

it can be deduced that for a flat sample the electrode charges $\sigma_{1}$ and $\sigma_{2}$ are given by

$$
\begin{gathered}
\sigma_{1}=\varepsilon_{0} \varepsilon_{r} E_{1}, \\
\sigma_{2}=-\varepsilon_{v} \varepsilon_{r} E_{2},
\end{gathered}
$$

where $E_{1}$ stands for the magnitude of the electric field at $E l_{1}$ and $E_{2}$ stands for the magnitude of the electric field at $\mathrm{El}_{2}$.

\section{At the Electrodes}

Electrostatic forces per unit area, i.e., pressure, generated at the electrodes $E l_{1}$ and $E l_{2}$ are then given by

$$
\begin{aligned}
& p_{1}=\frac{1}{2} \varepsilon_{0} \varepsilon_{r} E_{1}^{2}=\frac{1}{2} \sigma_{1} E_{1}, \\
& p_{2}=-\frac{1}{2} \varepsilon_{0} \varepsilon_{r} E_{2}^{2}=\frac{1}{2} \sigma_{2} E_{2} .
\end{aligned}
$$

We introduce a total field comprised of two parts; the dc field $E_{i, D C}$ and the pulse field $E_{p}$ (which is approximated by $U_{p} / d_{\text {sample }}$ ) by

$$
\begin{aligned}
& E_{1}=E_{1, D C}+E_{p}, \\
& E_{2}=E_{2, D C}+E_{p} .
\end{aligned}
$$

It follows that

$$
\begin{gathered}
p_{1}=\frac{1}{2} \varepsilon_{0} \varepsilon_{r}\left(E_{1, D C}^{2}+E_{p}^{2}+E_{1, D C} E_{p}\right), \\
p_{2}=-\frac{1}{2} \varepsilon_{0} \varepsilon_{r}\left(E_{2, D C}^{2}+E_{p}^{2}+E_{2, D C} E_{p}\right) .
\end{gathered}
$$

The term $E_{i, D C}{ }^{2}$ represents a constant pressure that can be neglected. By using (2) and (3) we end up with

$$
\begin{aligned}
& p_{1}=\sigma_{1, D C} E_{p}+\frac{1}{2} \varepsilon_{0} \varepsilon_{r} E_{p}^{2} \\
& p_{2}=\sigma_{2, D C} E_{p}-\frac{1}{2} \varepsilon_{0} \varepsilon_{r} E_{p}^{2}
\end{aligned}
$$

Generally $E_{p}{ }^{2}<<\sigma_{3, D C} E_{p}$. Therefore, it follows that

$$
\begin{aligned}
& p_{1} \approx \sigma_{1, D C} E_{p}, \\
& p_{2} \approx \sigma_{2, D C} E_{p} .
\end{aligned}
$$

\section{Within the Dielectric Bulk}

The electric pulse $E_{p}$ with width $\Delta T$ acts on a slab of space charge of width $b=v_{s a} \Delta T$, where $v_{s a}$ denotes the speed of sound inside the sample. Then the pressure at place $x$ inside the sample is given by

$$
p_{3}(x)=\rho(x) b E_{p} .
$$

The description has used the electrostatic approximation, which in practice has proved to be sufficient.

It results from (12) and (13) that the amplitude of the pressure waves originating from the electrode charges depend linearly on the pulse voltage and the electrode charges themselves. From (14) it follows that the pressure amplitude originating from the space charge depends linearly on the pulse voltage and the space charge itself.

\section{Acoustic Description}

Every layer in the measurement system (Fig. 2) has a certain, known acoustical impedance $Z_{x}$. When there is a mismatching of these impedances at two successive layers, reflection and transmission of the acoustic waves will occur at the interfaces. From each charge source, i.e., the electrodes $E l_{1}$ and $E l_{2}$ and the space charges inside the sample, only a part of the acoustic wave will arrive at the transducer

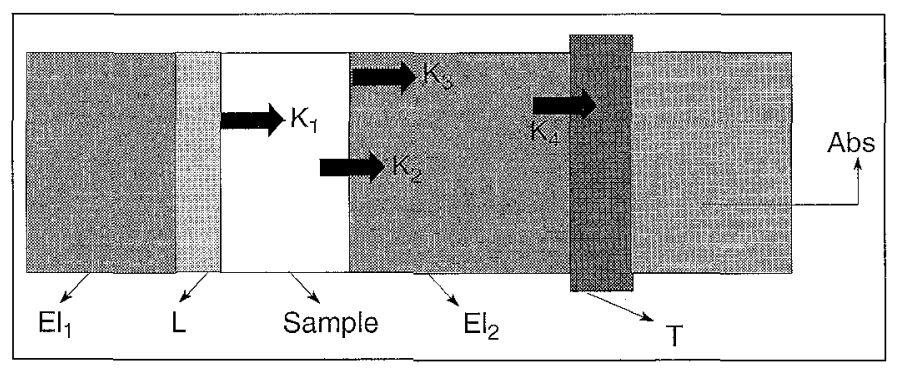

Fig. 2 Acoustic description of the measurement set-up 
$T$ at one moment in time. This can be described by introducing transmission coefficients $K_{x}$.

The part of the acoustic wave originating from $E l_{1}\left(p_{1}\right)$ that arrives at transducer $T\left(p_{1, T}\right)$ is given by

$$
p_{1, T}=K_{1} K_{2} K_{4} p_{l} \text {. }
$$

The part of the acoustic wave originating from $E l_{2}$ that arrives at transducer $T$ is given by

$$
p_{2, T}=K_{3} K_{4} p_{2} .
$$

The part of the acoustic wave originating from a slab of space charge that arrives at transducer $T$ is given by

$$
p_{3, T}=\frac{1}{2} K_{2} K_{4} p_{4},
$$

where

$$
\begin{gathered}
K_{1}=\frac{Z_{s a}}{Z_{s a}+Z_{L}} \quad K_{2}=2 \frac{Z_{\text {Aluminum }}}{Z_{\text {Aluminum }}+Z_{s a}} \\
K_{3}=\frac{Z_{\text {Aluminum }}}{Z_{\text {Aluminum }}+Z_{s a}} \quad K_{4}=2 \frac{Z_{T}}{Z_{T}+Z_{\text {Aluminum }}},
\end{gathered}
$$

and $Z_{x}$ stands for the acoustic impedance of material $x$.

For an equal weighing of the acoustic waves, which in turn is necessary for an equal weighing of the different sources of space charges it is necessary that

$$
K_{1} K_{2} K_{4}=K_{3} K_{4}=\frac{1}{2} K_{2} K_{4} \text {. }
$$

Equation (20) will be satisfied if (using (18) - (21)),

$$
Z_{s a}=Z_{L} \text {. }
$$

Layer $L$ may be chosen to have the same acoustic impedance as the sample material and may be conductive for easy contact to the sample. Without this layer, the acoustical interface would be formed by the sample material and the aluminum electrode $E l_{1}$. The acoustical impedance of aluminum (and in fact of any useful metal), however, is approx. ten times higher than the most used plastic samples like PE, LDPE, HDPE, PMMA, etc., so that condition (21) would not be satisfied.

\section{Calibration}

The output signal $u_{\text {osc }}$ in Volts on the oscilloscope must be translated into $\mu \mathrm{C} / \mathrm{cm}^{3}$. By using equation (14), which is valid for charge density $\left(\mu \mathrm{C} / \mathrm{cm}^{3}\right)$, we can write

$$
u_{\text {osc }}=\kappa p=\kappa E_{p} b \rho=\mathrm{K} \rho,
$$

where $\mathrm{K}$ is the overall calibration factor, which, in this survey, is independent of frequency. To be more complete (and sometimes necessary!) the full frequency dependent response function of transducer and amplifier should be taken into account.

If the sample is initially free of any space charge, putting a $\mathrm{dc}$ voltage across it has the effect of creating only electrode charges $\sigma_{1}$ and $\sigma_{2}$, but no space charges. The dc voltage must therefore be chosen not too high so that no space charge will be injected. Under these conditions we may calculate the electrode charges $\sigma_{1}$ and $\sigma_{2}$ by using (2) and (3)

$$
\sigma_{c a l}=\varepsilon_{0} \varepsilon_{r} \frac{U_{D C}}{d},
$$

where $\sigma_{c a l}$ stands for the electrode charges of the sample without space charges. Together with (12) and (13) we arrive at

$$
p_{c a l}=\frac{\varepsilon_{0} \varepsilon_{r} U_{D C} E_{p}}{d}
$$

where $p_{c a l}$ stands for the pressure originating from the electrode during calibration.

Combining (22)-(24) we arrive at an expression for $K$,

$$
K=\frac{d b}{\varepsilon_{0} \varepsilon_{r}} \frac{u_{o s c, c a l}}{U_{D C}}
$$

where $u_{o s, c a l}$ denotes the oscilloscope signal during calibration. When the dc voltage $U_{D C}$ or the pulse voltage $U_{p}$ is changed during a measurement, a new calibration factor $K$ must be determined. A more elaborate discussion about calibration, including a convolution type description of the signals may be found in [14]. For the sake of explaining the principle of calibration, we have given a simplified expression.

\section{System Response}

It is important to have a good understanding of the frequency behavior of transducer $T$ and amplifier $A$ as in some cases they can distort the acoustic wave. As a result, part of the output signal may be wrongly interpreted as space charge, but it is in fact a result of the frequency response of the transducer and amplifier. The distortion takes place, for instance, if the transducer and amplifier do not pass the total frequency content of the original acoustic signal.

Distortion takes place if the frequency response of the amplifier is not flat, if the amplifier's high cut-off frequency is too low, or if the combination of transducer and amplifier act as a high-pass filter. The first two problems are easily solved by using a high quality amplifier with good and sufficient frequency characteristics. The third problem deserves some extra attention.

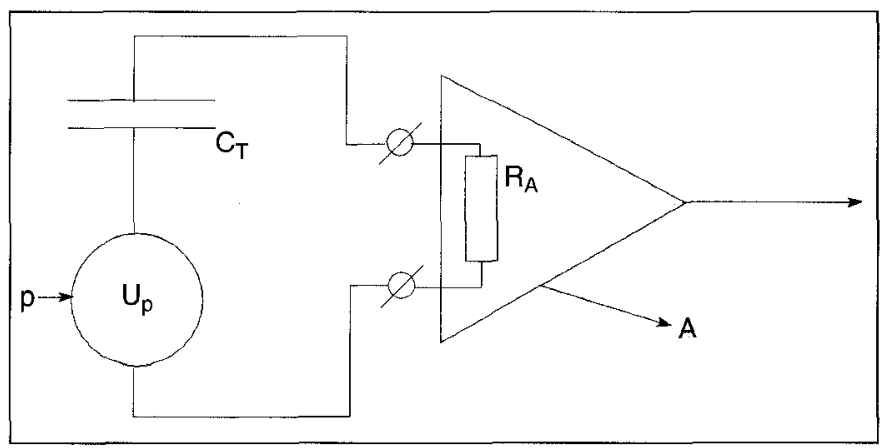

Fig. 3 Circuit description of the transducer amplifier combination. The PVDF sensor is represented by a frequency independent pressure $p$ to voltage $U_{p}$ transformer. 


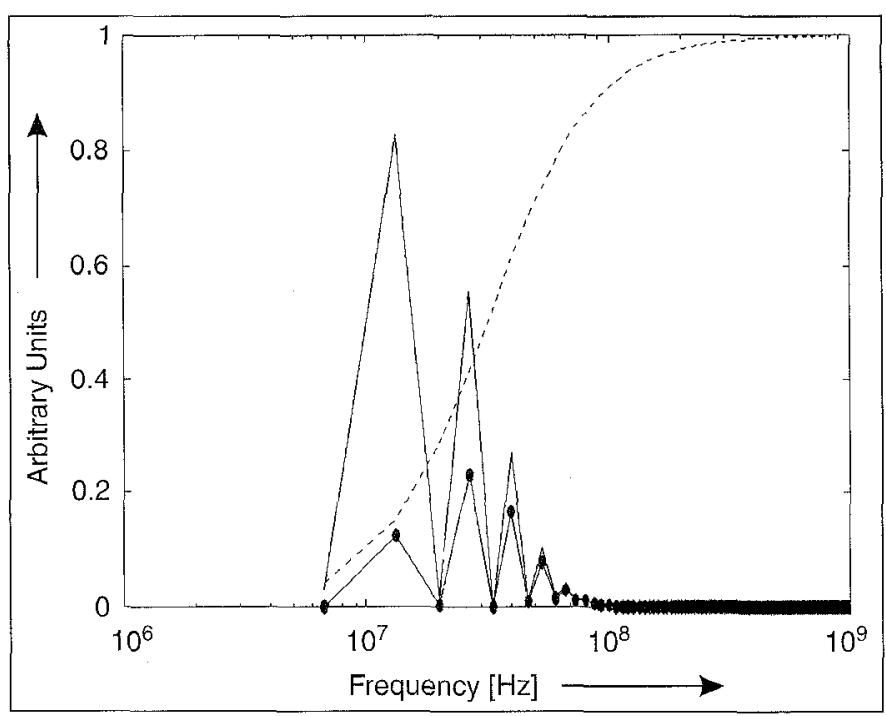

Fig. 4 Frequency characteristic $W(f)$ of transducer/amplifier combination (dash-dot line, $R_{A}=50 \Omega, C_{T}=0.1 \mathrm{nF}$ ), frequency characteristic of original signal $P(f)$ (solid line) and frequency characteristic $V(f)$ of the output signal (solid line with circles)

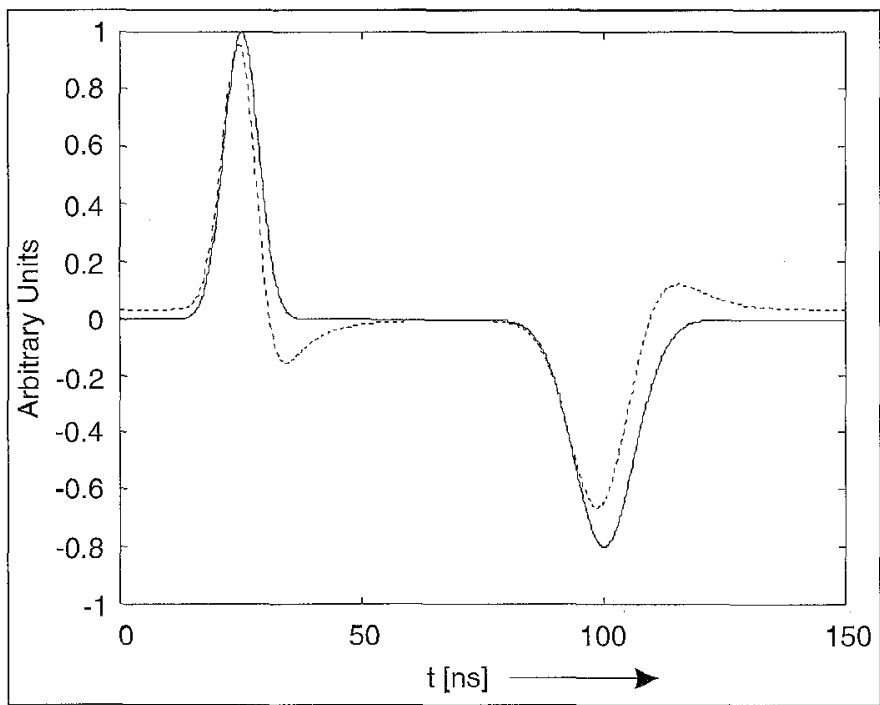

Fig. 5 Time domain signals. The simulated acoustic signal $p(t)$ (solid line) and the distorted signal $v(t)$ (dash-dot line)

The transducer consists of a PVDF sensor that has a high capacitance (typically 0.1-5 nF). Low-noise and highfrequency amplifiers $(500 \mathrm{MHz})$ often have a low input impedance $(50 \Omega)$ (see Fig. 3 for the simplified circuit description). Together, they act as a high pass filter with a cut-off frequency that may lie well in the range of the frequency content of the acoustic signal.

The frequency response function of the circuit is given by

$$
W(f)=\frac{2 \pi j f C_{T} R_{A}}{1+2 \pi j f C_{T} R_{A}},
$$

where $R_{A}$ denotes the input impedance of amplifier $A, C_{T}$ denotes the capacity of the transducer $T, f$ stands for the frequency of the signal, and $j$ is the imaginary unit.

The frequency plot of W(f) is plotted in Fig. 4 . In the following we will simulate what will happen with an undis- torted acoustic signal when it is detected by the transducer/amplifier combination. In Fig. 5 the simulated acoustic signal $p(t)$ is plotted (resulting from a 10 -ns-wide electrical pulse). This is the original undistorted signal. The signal $p(t)$ is transformed into the frequency domain by using an FFT algorithm, which gives us $P(f)$, which is plotted in Fig. 4. The frequency domain voltage output signal $V(f)$ is calculated by

$$
V(f)=P(f) W(f)
$$

As may be seen in Fig. 4, the lower frequency content of the original signal $P$ is attenuated by the frequency response $W(f)$ of the transducer/amplifier combination. When we transform $V(f)$ back into the time domain by using an inverse FFT algorithm, we arrive at a distorted signal $\nu(t)$ that may be found in Fig. 5. The negative undershoot of the first pulse is not the result of real measured space charge but of the frequency response of the measurement system. This result should not be misinterpreted! This problem can be solved mathematically or by using an amplifier with an higher input impedance, for instance $50 \mathrm{k} \Omega$. The disadvantage of such amplifiers is that their noise level is much higher.

We only took the $R_{A} C_{T}$ distortion into account. To be more correct, the frequency-dependent response function of the PVDF sensor and of the sample material itself should be taken into account. Whether one should use elaborate convolution and FFT techniques to restore the original signal or not depends on the desired accuracy and the purpose of the measurement. The result stresses the fact that the PEA measurement system and its frequency response must be known before an interpretation of signals can take place.

\section{Test Results on Impregnated Paper}

Many tests have been performed by other authors on polymeric samples. Little information, however, exists on testing mass-impregnated paper insulation. High Voltage Direct Current (HVDC) submarine cables are mostly of the MIND (Mass Impregnated Non-Draining) type and use this

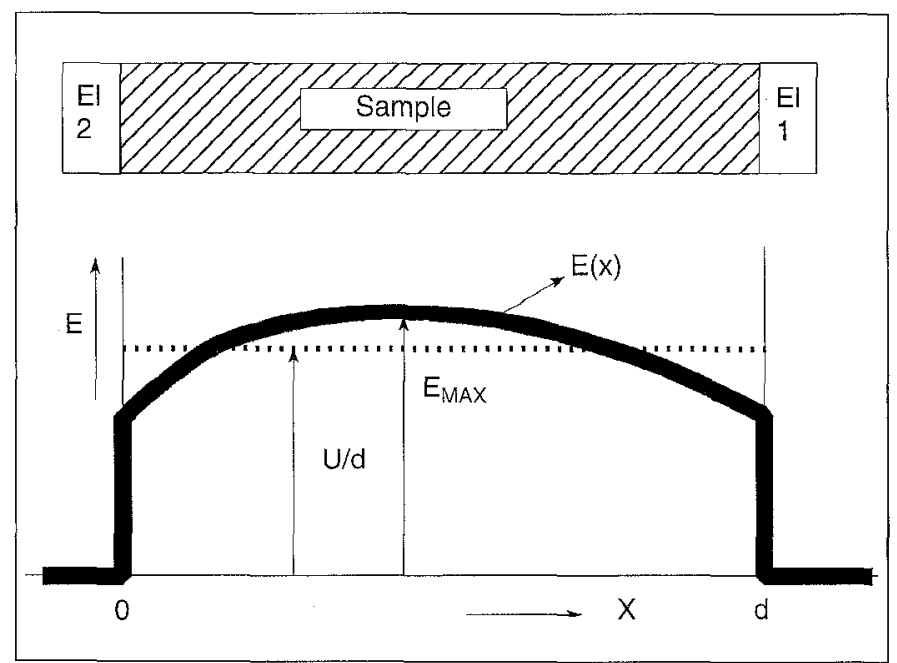

Fig. 6 Definition of electric field symbols 


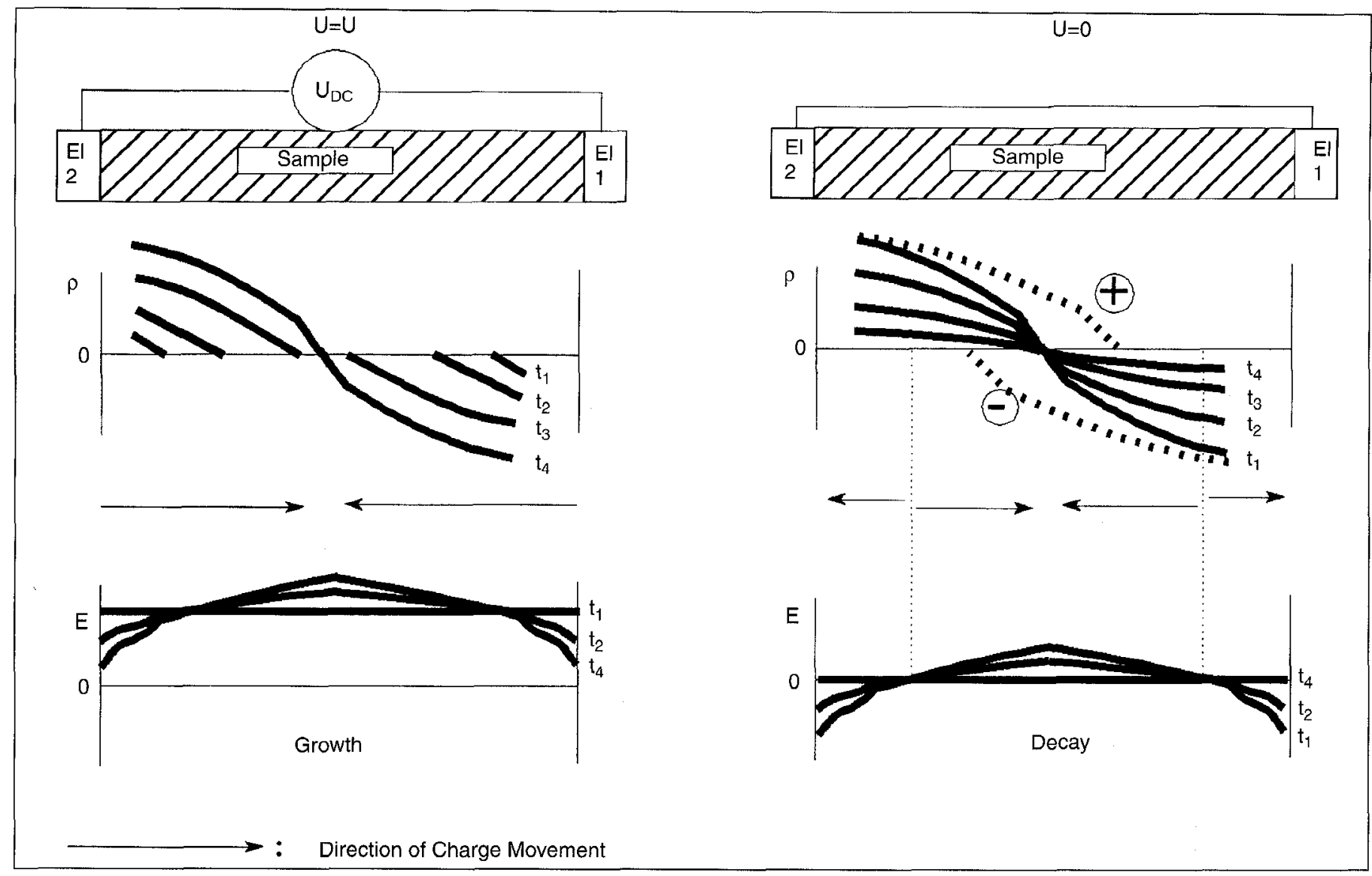

Fig. 7 Difference in growth and decay patterns. Charge density profiles $\rho$ and electric fields $E$ are shown. The figures are stylized.

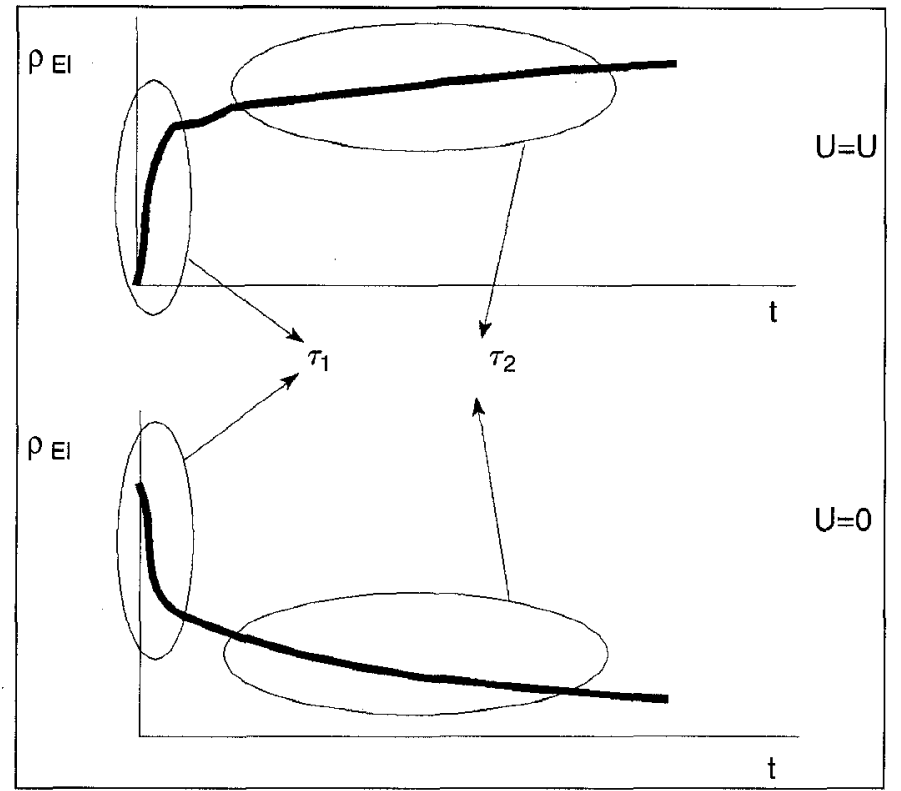

Fig. 8 The observation of two time constants. A small one $\tau_{1}$ and a large one $\tau 2$. PEL stands for the maximum charge density near an electrode.

kind of paper insulation. As these cables are often part of very large projects [15] and as they have proven to be very reliable, it is interesting to investigate the space charge behavior of this type of insulation.
The tests were performed using flat paper samples instead of full-size cables. In the first approximation, it was assumed that the space charge behavior of a flat paper sample is not different from the behavior of a paper stroke in a ready cable. Therefore, in the first approximation, it was considered justifiable to test paper samples instead of fullsize cables.

Different types of paper and oil combinations have been tested. The papers differed mainly in thickness $(100-155 \mu \mathrm{m})$ and air impermeability (100-10000 Gurley seconds), whereas the oil differed mainly in resistivity $(10-400 \mathrm{~T} \Omega \mathrm{m})$ and viscosity $\left(4-7500 \mathrm{~mm}^{2} / \mathrm{s}\right)$. The samples were prepared in such a way, that the production process of full size cables was reflected as much as possible. The paper was first vacuum dried at $120^{\circ} \mathrm{C}$, and after that, it was impregnated at $120^{\circ} \mathrm{C}$. The temperature of the samples was then reduced by natural cooling.

The samples were kept in glass bottles. The oil level in the bottle was such that the paper was immersed in the oil completely. The bottle was wrapped in aluminum foil. These measures were taken to prevent air and moisture from diffusing into the sample.

It was concluded from the test results that whatever the combination of paper and oil, three general trends were observed. They will be mentioned first and discussed later on.

1. Homocharge is always observed, both at anode and cathode.

2. A specific charge growth/decay pattern occurs. 


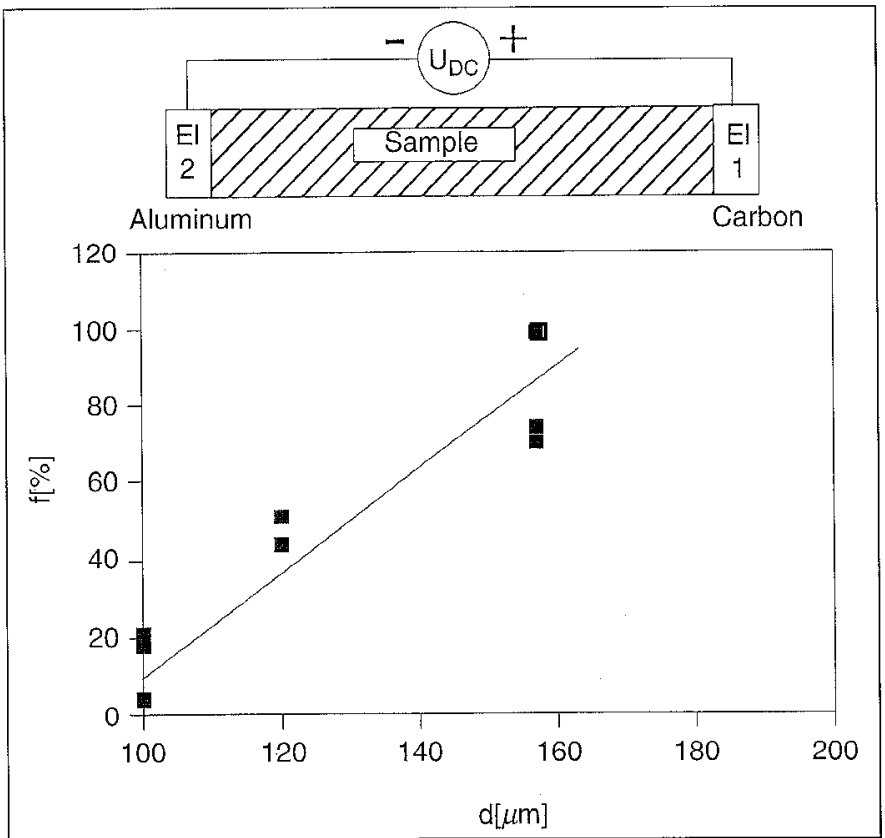

Fig. 9 Field enhancement factor $f$ as a function of paper thickness $d$

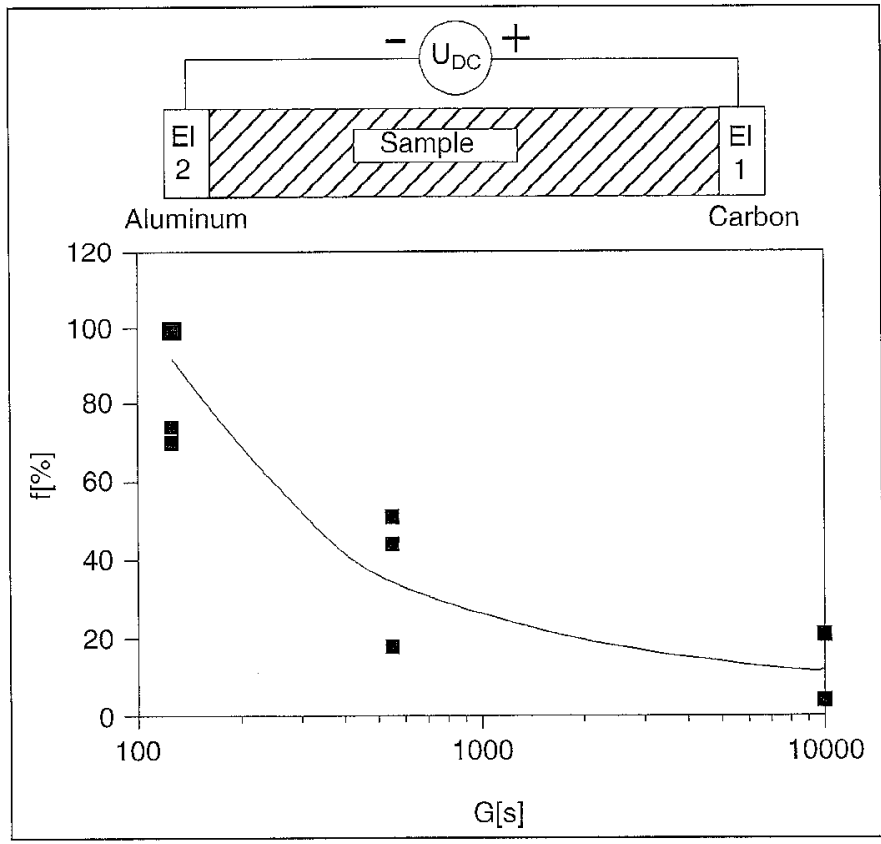

Fig. 10 Field enhancement factor $f$ as a function of air impermeability $G$

3. The growth and decay of the charge profiles can be described by roughly two time constants, a small one (order of magnitude 1 minute) and a large one ( $>1 / 2$ hours).

The observations were done using both voltage polarities and different voltages $(2-8 \mathrm{kV})$. The two different electrode materials that have been used, conducting paper and aluminum, had no influence on these general trends.

\section{Homocharge}

The fact that homocharge occurs in all cases means that interface processes dominate over the bulk conduction mechanism(s). Charges are more easily injected than they at transported away from the electrode.

The maximum of the charge densities at a moderate homogeneous dc stress $(U / d)$ of $20 \mathrm{kV} / \mathrm{mm}$ varied between roughly 10 and $30 \mathrm{C} / \mathrm{m}^{3}$.

The high reproducability of homocharge patterns in paper is quite remarkable as space charge distributions in plastic samples or PE cables are usually hardly reproducable $[1,16]$.

If homocharge appears at both electrodes, it results in a field enhancement in the middle of the paper (see Fig. 6). The relative field increase as compared with the homogeneous dc field will be called the field enhancement factor $f$ and is defined as

$$
f=\frac{E_{\operatorname{MAX}}-\frac{U}{d}}{\frac{U}{d}} \cdot 100 \%,
$$

in which $E_{M A X}$ is the maximum field strength in the sample, $U$ is the external voltage and $d$ is the thickness of the sample.

\section{Growth/Decay Pattern}

The difference in growth and decay patterns is shown in Fig. 7. Starting from an initial space charge- free sample, it was observed that the growth of charge starts from near the electrodes. After some time the charge grows in magnitude and travels deeper into the paper. When the positive and negative homocharge meet each other in the middle of the paper, a stable situation occurs. It is assumed that recombination of charge carriers takes place in this region.

When removing the voltage, the charge profile changes in magnitude only; the homocharges diminish.

This is explained by the fact that the field direction in the middle of the paper stays the same after removal of the voltage. As a result, the charges still move to each other until complete recombination has taken place. Nearer the electrodes, the field direction is reversed due to the removal of the voltage. The charges in this region will travel back toward the electrodes. It is assumed that they are adsorbed there.

\section{Time Constants}

The growth and decay of space charge can be described by two time constants (see Fig. 8). The first time constant is a short one ( $<1$ minute). The second one, although it differs much per sample, mostly is $>1 / 2$ hours. It is explained as follows.

Charges are injected at the electrodes in an initially space charge-free sample. This may happen quite quickly and is described by the first time constant.

Injection processes are often field-dependent in a nonlinear way (think of the Schottky injection, for instance). The higher the interface field, the higher the rate of charge injection. As a homocharge is formed, the interface field is lowered, thus decreasing the rate of injection. After the injection, the charges will move deeper into the sample and will prevent the homocharge profile from stabilizing immediately. Only after the three processes (injection, transportation, and recombination) are in balance does the 


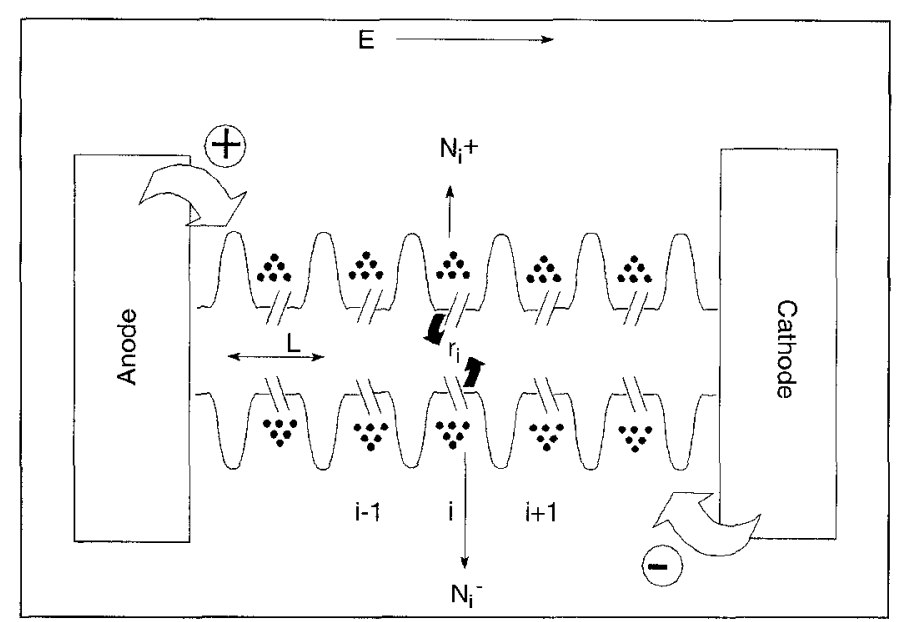

Fig. 11 Space charge model as applied to impregnated paper. $r_{i}$ stands for the rate of recombination at place $i, N_{i}$ stands for the carrier density at place $I$, and $L$ stands for the distance between two successive energy barriers.

homocharge profile become stable. These combined actions may then be described by the second time constant.

\section{The Effect of Paper and Oil Properties on the Field Enhancement Factor}

The field enhancement factor $f$ may be seen as a paper perfomance factor. The higher the field enhancement factor, the higher the field in the middle of the paper, which is not desirable. And even worse, after a polarity reversal, the field at the paper interface is increased. Interfaces are generally the weakest points in an insulation system. It is therefore desirable to keep the field enhancement factor $f$ as low as possible.

In Fig. 9, the field enhancement factor $f$ is shown as a function of the paper thickness $d$, and in Fig. 10, $f$ is shown as a function of the air impermeability $G$. It is concluded that the lower the thickness $d$ and the higher the air impermeability $G$, the lower the field enhancement factor $f$.

There were no paper samples available such that the thickness and the air impermeability of the samples could be changed independently. At this stage it is therefore more correct to evaluate the combined quantity $\{d, G\}$ and not to evaluate the quantities separately. It is therefore concluded that thin paper with a high air impermeability coefficient is the best choice to keep the field enhancement low.

From other observations, it was concluded that the higher the conductivity $\sigma$ the lower the field enhancement factor $f$. The results are not shown here.

The table shows what type of paper and oil should be used in order to keep the field enhancement factor low.

\begin{tabular}{|l|l|l|l|}
\hline \multicolumn{4}{|c|}{ Table I } \\
\hline \multirow{4}{*}{ property } & paper & air impermeability & conductivity \\
\cline { 2 - 4 } & thickness & high & high \\
\hline choice & small &
\end{tabular}

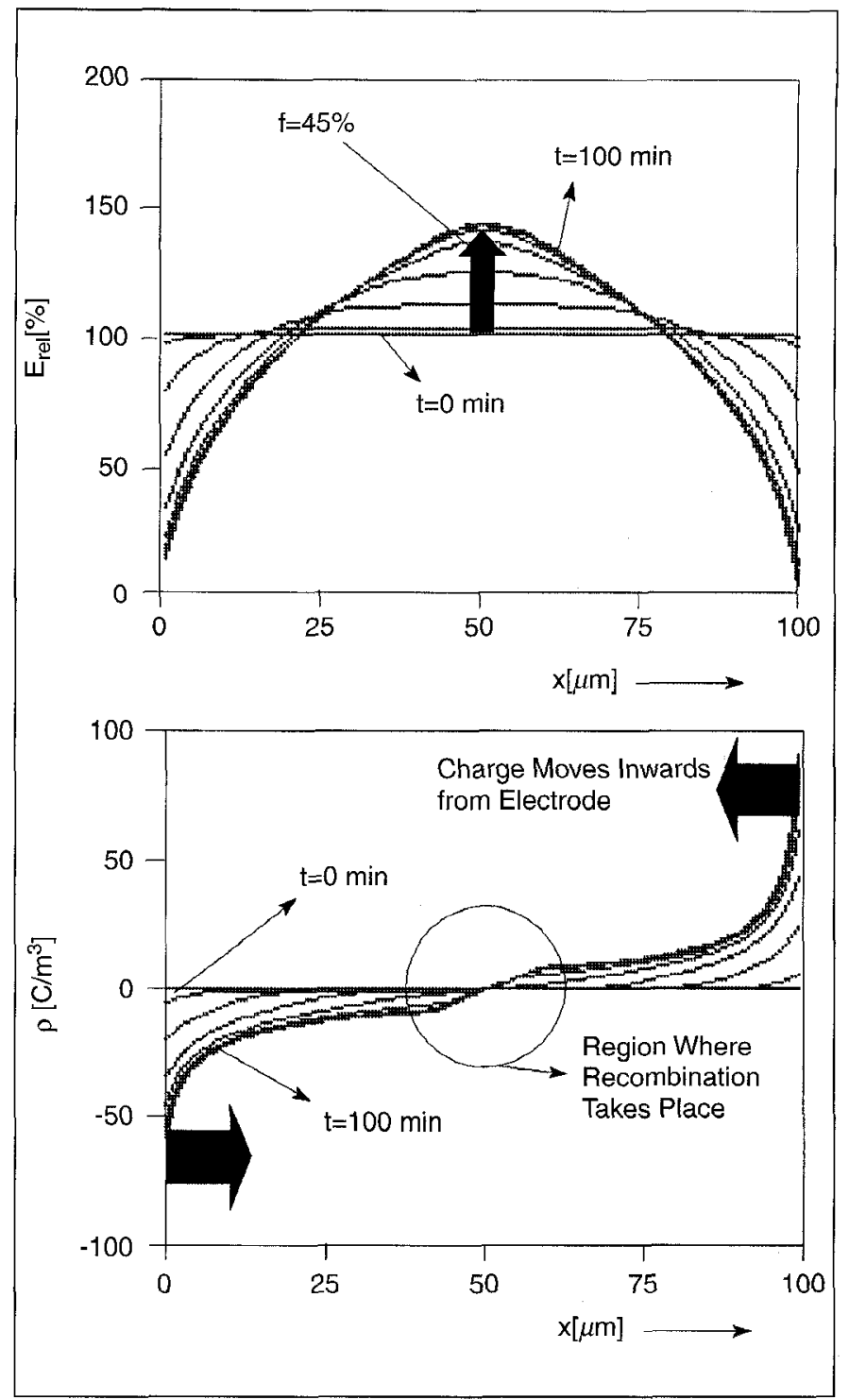

Fig. $12 \mathrm{~A}$ simulation result of a growth pattern. The voltage during simulation was $2 \mathrm{kV} \mathrm{dc}$.

\section{A Physical Model for Space Charge Accumulation in Impregnated Paper}

A model has been built, based on ionic conduction [17], Schottky injection [18], and recombination. The model predicts the occurrence of homocharge, the specific growth/decay patterns, and the order of magnitude of the charge density and the field enhancement factor. However, it failed to predict the occurrence of the two time constants.

Fig. 11 shows the principle of the model. The ionic conduction in the bulk of the paper is modeled as energy barriers placed in a row at a successive distance $L$, which later on proved to be in the order of magnitude of $0.1-5 \mu \mathrm{m}$ (comparable to the pore structures in paper). The energy barriers have been set up for both positive and negative charge carriers. A Schottky injection of positive charges has been modeled at the anode and a Schottky injection of negative charge carriers at the cathode. Recombination was modeled 
throughout the whole sample and was made a function of the charge carrier density as a function of location.

The different parameters of the ionic conduction model and the Schottky model have been chosen, based on steadystate conduction considerations [17]. Other parameters, such as the rate of recombination and the distance between the energy barriers, have been chosen in an iterative way in order to match the observed values of charge density $\rho$, field enhancement factor $f$ and time constant $\tau_{2}$ (the large one).

Fig. 12 shows a result of the simulation of the growth of the charge. The lower part of the figure shows the charge density $\rho$ as a function of location, whereas the upper part shows the field $E_{\text {rel }}$ relative to the Laplacian field in percentages.

The values of field enhancement, homocharge density, and time constant agree very well with the observed values within the order of magnitude. The specific pattern of charge growth is also clearly observable: charges start at the electrode and move to the interior of the paper.

The region very close to the electrodes (first $20 \mu \mathrm{m}$ or so) could not be observed in practice, due to the limited resolution of the measurement system. The charge densities as measured may therefore be compared only with the calculated values, disregarding the regions very close to the electrodes.

The calculated decay pattern (not shown here) corresponded with the observations, too.

A more detailed evaluation about the testing and the discussion on space charge measurements on impregnated paper will be given in a paper that will be presented elsewhere.

\section{Conclusions}

Together with the Laser Induced Pressure Pulse (LIPP) method, the electrically pulsed acoustic method (PEA, ESAW) is the most commonly used principle of measuring space charge nowadays. The PEA measurement method was described against the background of the historical search for a non-destructive way of measuring space charge in a quantitative manner. An electrostatic approximation was used to describe the principle of the electrically pulsed method. Either the acoustic impedances must be known or an acoustic layer at the high voltage electrode must be used in order to quantify the surface charges on that electrode in a correct manner. For some transducers, the charge signal will be distorted by the combination of transducer and amplifier when using a low input impedance $(50 \Omega)$ amplifier. It depends on the desired accuracy and the purpose of the measurement whether one should use mathematical tools to reveal the original signal.

Test results of PEA measurements on oil-impregnated paper samples were presented. In most of the literature, space charge measurement results are shown for plastic samples. To the authors' best knowledge, this is the first time that results have been published on space charge measurements on HVDC cable paper.

Thick papers with a low air impermeability coefficient suffer from the highest field enhancements in the middle of the paper. Regarding the oil, the conductivity should not be chosen too low, in order to keep the field enhancement factor low. A physical model based on ionic conduction, Schottky injections of both positive and negative charge carriers, and recombination was able to predict most of the observations.

\section{Acknowledgments}

The authors wish to express special thanks to Prof. T. Takada of the Musashi Institute of Technology for his advice and the valuable discussions regarding the Pulsed Electroacoustic method.

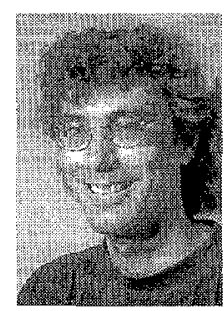

Peter Morshus studied at the Delft University of Technology and obtained his Ph.D. in electrical engineering in 1993 on ultrawide band optical and electrical analysis of partial discharge ageing. From 1986 to 1988 he worked for the High Voltage Research Laboratory of NKF Kabel in Delft, where he studied the effect of defects on cable life. Since 1988 he has been an assistant professor at the High Voltage Laboratory of the Faculty of Electrical Engineering of Delft University of Technology. He is responsible for the fields of material ageing and HVDC in a number of projects and works in close cooperation with industry. He may be reached at: High Voltage Laboratory, Delft University of Technology, Mekelweg 4, 2628 CD Delft, The Netherlands.

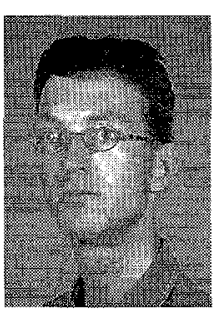

MARC Jeroense studied at the Advanced School of Technology in Vlissingen from 1984 to 1988. He finished at Delft University of Technology in 1992. In 1992 he joined the High Voltage Research Laboratory of the cable factory, NKF Kabel in Delft, and he is in the midst of a Ph.D. program on HVDC cables at Delft University of Technology.

\section{References}

1. M.J. R. Jeroense and F.H. Kreuger, "Wanneer $50 \mathrm{~Hz}$ niet meer helpt.....DC! De juiste verbinding,"

Energietechniek, pp. 457-460, July/August, 1995.

2. F.H. Kreuger, Industrial High DC Voltage, Delft University Press, 1995

3. U. Fromm, Partial Discharge and Breakdown Testing at High DC Voltage, Diss., TU Delft, Delft University Press, 1995.

4. Y. Toriyama, Dust Figure Of Surface Discharge and Its Application, Kinokuniya Bookstore Co. LTD., Tokyo, 1961.

5. Y. Murooka and K. Hidaka, "Theoretical Studies on Nanosecond Surface Discharge Phenomena Observed Using Lichtenberg Figure Method," Arch. Electrotech., V. 74, pp. 163-173, 1990.

6. T. Jing, Surface Charge Accumulation in $S F_{6}$, Diss., TU Delft, Delft University Press, 1993

7. D.K. Davies, "Examination of the Electrical Properties of Insulators by Surface Charge Measurements," J. Sci. Instrum., V. 44, pp. 521-524, 1967. 8. A. Toureille, "About a Method to Measure Space Charge Density Inside PE," Jicable 87, Sec. Int. Conf. on Polym. Insul. Power Cables, Versailles, pp. 98-103, 1987

9. A. Tanaka, M. Maeda, and T. Takada "Observation of Charge Behavior in Organic Photoconductor Using Pressure-Wave Propagation Method," IEEE Trans. on EI, Vol. EI-27, No. 3, pp. 440-444, 1992.

10. R. Gerhard-Multhaupt, "Analysis of Pressure-Wave Methods for the Nondestructive Determination of Spatial Charge or Field Distributions in Dielectrics," Phys. Rev. B, Vol. 27, No. 4, pp. 2494-2503, 1983.

11. J. Lewiner, "Evolution of Experimental Techniques for the Study of the Electrical Properties of Insulating Materials," IEEE Trans. on EI, Vol. EI-21, No. 3, pp. 351-360, 1986. 
12. T. Maeno, T. Takada, C.M. Cooke, et al., "Measurement of Spatial Charge Distribution in Thick Dielectrics Using the Pulsed ElectroAcoustic Method," IEEE Trans. on EI, Vol. EI-23, No. 3, pp. 433-439, 1988.

13. Y. Li and T. Takada, "Experimental Observation of Charge Transport and Injection in XLPE at Polarity Reversal," J. Phys. D: Appl. Phys., 25, pp. 704-716, 1992.

14. Y. Li, M. Yasuda, and T. Takada, "Pulsed Electroacoustic Method for Measurement of Charge Accumulation in Solid Dielectrics," IEEE Trans. on Diel. and El. Ins., Vol. 1, No. 2, pp. 188-195, 1994.
15. Sáiz Chicharro, "Comparison Between AC and DC Transmission," CIGRE SC 37, Report No. 37-94 (GT 12) 06 (E).

16. Y. Li, M. Yasuda, and T. Takada, "Influence on Spatial Charge Distribution of Cross-Linking Agent Residues in XLPE," Proc. of the $3^{\text {rd }}$ Int. Conf. on Prop. and Appl. of Diel. Mat., pp. 1210-1213, July 8-12, 1991.

17. M.J.P. Jeroense and F.H. Kreuger, "Electrical Conduction in HVDC Mass-Impregnated Paper Cable," IEEE Trans. on Diel. and EI, Vol 2, No. 5, October 1995

18. L.A. Dissado and J.C. Fothergill, Electrical Degradation and Breakdown in Polymers, Peter Peregrinus Ltd., London, 1992.

\section{Comparative Investigation}

\section{(continued from page 25)}

38. R. Bozzo, A. Guastavino, M. Cacciari, A. Contin, G.C. Montanari, "Stochastic Procedures for Investigation of Tree Growth in Insulating Materials for HV Applications," Proc. IEEE ISEI, pp. 54-57, Pittsburgh, USA, 1994.

39. G. C. Montanari, M. Cacciari, "Electrical Life Threshold Models for Insulating Materials Subjected to Electrical and Multiple Stresses. Part 2: Probabilistic Approach to Generalized Life Models," IEEE Trans. on El. Ins., Vol. 27, No. 5, pp. 1000-1008, October 1992.

40. E. Occhini, "A Statistical Approach to the Discussion of Dielctric Strength in Electric Cables," IEEE Trans. on P.A.S., Vol. 90, No. 6, pp. 2671-2678, December 1971.

41. S.L. Mc Cune, E.D. Mc Cune, M. Waiton, J.T. Smith III, B. Bernstein, W.A. Thue, P. Cox, R.L. Harp, E. Rogers, M. L. Walker, "A Statistical Aging Model for XIPE-Insulated Medium Voltage Distribution Cables," Proc. IEEE CEIDP, pp. 458-470, Arlington, USA, October 1994.

42. J. Biernat, J. Jarnicki, K. Kaplon, A. Kuras, G.J. Anders, "Reliability Considerations in Accelerated Life Testing of Electrical Insulation with
Generalized Life Distribution Function," IEEE Trans. on Power Sys., Vol. 7, No. 2, pp. 656-664, May 1992.

43. R.E. Kalman, "A New Approach to Linear Filtering and Prediction Problems," Trans. ASME, series D, Journal of Basic Engineering, Vol.82, pp. 35-45, March 1960.

44. M. Cacciari, G.C. Montanari, G. Tambini, "Inference Life Models of Electrical Insulating Materials Using a Kalman Filter," IEEE Trans. on Reliability, Vol. 43, No. 2, pp. 210-216, June 1994.

45. G.C. Montanari, G. Mazzanti, M. Cacciari, "Application of Kalman Filter for Electrical Endurance Characterization of Insulating Materials and Systems," IEEE Trans. on Diel. and El. Ins., Vol. 3, No. 1, pp. 56-63, February 1996.

46. M. Cacciari, G.C. Montanari, C.P. Barry, "Thermal Endurance of Electrical Insulating Materials Studied by the Use of the Kalman Filter," ETEP, Vol. 6, No. 2, pp. 103-110, April 1996. 